\title{
Photoinduced Deaminative Borylation of Unactivated Aromatic Amines Enhanced by $\mathrm{CO}_{2}$
}

\author{
Akira Shiozuka," Kohei Sekine, ${ }^{*, \#, \| l ~ Y o i c h i r o ~ K u n i n o b u *, \#, ॥ ~}$ \\ \#Institute for Materials Chemistry and Engineering, Kyushu University, 6-1 Kasugakoen, Kasuga-shi, Fukuoka 816- \\ 8580, Japan \\ "Interdisciplinary Graduate School of Engineering Sciences, Kyushu University, 6-1 Kasugakoen, Kasuga-shi, Fukuoka \\ 816-8580, Japan
}

KEYWORDS: C-N Bond Transformation, Borylation, Photocatalyst, Carbon Dioxide

\begin{abstract}
Herein, direct unactivated C-N borylation of aromatic amines by a photocatalyst was achieved under mild and metal-free conditions. The $\mathrm{C}-\mathrm{N}$ borylation of aromatic amines with bis(pinacolato)diboron $\left(\mathrm{B}_{2} \mathrm{pin}_{2}\right.$ ) proceeded in the presence of a pyrene catalyst under light irradiation $(\lambda=365 \mathrm{~nm})$ to afford desired borylated products and aminoborane as a byproduct. The yield of borylated product improved under a $\mathrm{CO}_{2}$ atmosphere. Reactions conducted in the presence of a stoichiometric amount of aminoborane under $\mathrm{N}_{2}$ or $\mathrm{CO}_{2}$ indicated that $\mathrm{CO}_{2}$ reduced the inhibitory effect of aminoborane. Optimal reaction conditions were applied to a variety of aromatic amines. Mechanistic studies suggested that the C-N bond cleavage and C-B bond formation proceeded via a concerted pathway.
\end{abstract}

Transformation via C-N bond cleavage is a promising protocol for synthetic applications, since a wide variety of natural and industrial products contain amino groups. In particular, arylamine moieties are common among bioactive natural products, pharmaceuticals, and organic functional materials. However, cleavage of aromatic $\mathrm{C}-\mathrm{N}$ bonds is challenging owing to their high bond dissociation energies. ${ }^{1}$ To overcome this problem, several strategies for $\mathrm{C}-\mathrm{N}$ bond transformation have been developed. Typically, highly reactive species, such as quaternary ammonium salts ([Ar$\left.\left.\mathrm{NR}_{3}\right]^{+}\right)$and diazonium salts $\left(\left[\mathrm{Ar}-\mathrm{N}_{2}\right]^{+} \mathrm{X}^{-}\right)$, are prepared from arylamines in advance or in situ, and their $\mathrm{C}-\mathrm{N}$ bonds are consequently converted to various functional groups (Scheme 1, a-1). ${ }^{2-5}$ The C-N bonds of Katritzky pyridinium salts, which are readily prepared from anilines, are also transformed by a photoredox or Lewis base catalyst (Scheme 1, a-1). ${ }^{6-9}$ For other methods, nickel-catalyzed reduction and borylation of $N$-aryl amides ${ }^{10}$ and coupling reactions of $\mathrm{N}$-arylsulfonamides ${ }^{11}$ and $\mathrm{N}$-aryl amides ${ }^{12}$ via C$\mathrm{N}$ bond cleavage were developed (Scheme 1, a-2). In these reactions, the $\mathrm{C}-\mathrm{N}$ bonds are more reactive than those of the corresponding amino groups because of the electron-withdrawing effect of the acyl or sulfonyl groups. Furthermore, direct transformations of unreactive aromatic C-N bonds have been investigated. ${ }^{13-19}$ For example, Kakiuchi et al. have recently developed directing-group-assisted ruthenium-catalyzed C-N bond functionalization of $\mathrm{N}, \mathrm{N}$-dimethyl aniline derivatives, and they reported hydrodeamination, alkyldeamination, and coupling reactions with the arylboronic acid ester of $o$-acylanilines (Scheme 1, b-1) ${ }^{13-15}$ In a similar study, Zeng et al. reported arylation and alkylation of arylamines via chromium-catalyzed $\mathrm{C}-\mathrm{N}$ bond cleavage, with imino groups as directing groups (Scheme 1, b-1). ${ }^{16,17}$ Although effective direct transformations of aromatic C-N bonds were achieved in the abovementioned reaction systems, they can only be applied to substrates with a directing group. The first example of $\mathrm{C}-\mathrm{N}$ transformation without the use of a directing group was reported by Shi et al. (Scheme 1 , b-2). They developed Ni-catalyzed direct C-N borylation ${ }^{20}$ and arylation ${ }^{21}$ of tertiary anilines. Direct $\mathrm{C}-\mathrm{N}$ transformations have generally been catalyzed by transition metal complexes via oxidative addition of the $\mathrm{C}-\mathrm{N}$ bonds to the metal center. ${ }^{22,23}$ However, metal-free direct transformations of unactivated aromatic $\mathrm{C}-\mathrm{N}$ bonds are yet to be developed.

Based on the above research background, we focused on a metal-free direct C-N borylation, because it is a powerful protocol for various organic syntheses. ${ }^{24,25}$ The resulting C$B$ bonds can be transformed into diverse substituents and functional groups. ${ }^{26}$ In this study, we report the first example of a metal-free direct $\mathrm{C}-\mathrm{N}$ borylation of unactivated aromatic amines by a photocatalyst under UV irradiation (Scheme 1c). In this reaction, readily available pyrene served as a photocatalyst, ${ }^{27,28}$ and a significant effect of $\mathrm{CO}_{2}$ on the efficient promotion of $\mathrm{C}-\mathrm{N}$ borylation was demonstrated. 
Scheme 1. Transformations via Cleavage of C-N Bond in Aromatic Amines

(a) Transformations of activated $\mathrm{C}-\mathrm{N}$ bonds

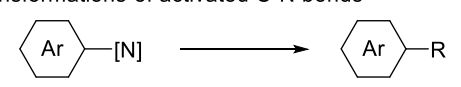

$$
[\mathrm{N}]= \begin{cases}\text { (a-1) salts } & \\ -\stackrel{+}{N} R_{3} \underline{X} & \text { ammonium salts } \\ -\stackrel{+}{N}_{2}- & \text { diazonium salts } \\ -N_{-}^{+} & \text {Katritzky pyridinium salts }\end{cases}
$$

(a-2) $N$-amides

$[\mathrm{N}]=\xi-\mathrm{NHTf}$

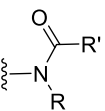

$(\mathrm{Tf}=$ trifluoromethanesulfonate $)$

(b) Transformations of unactivated $\mathrm{C}-\mathrm{N}$ bonds using transition metal catalysts (b-1) With directiong group

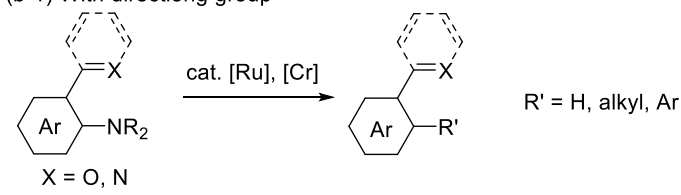

(b-2) Directing group-free

$$
\left\langle\mathrm{Ar}-\mathrm{NR}_{2} \stackrel{\text { cat. [Ni] }}{\longrightarrow \mathrm{Ar}-\mathrm{R}^{\prime} \quad \mathrm{R}^{\prime}=\text { Bnep, } \mathrm{Ar}}\right.
$$

(c) This work: Transformations of unactivated $\mathrm{C}-\mathrm{N}$ bonds without using transition metal catalyst

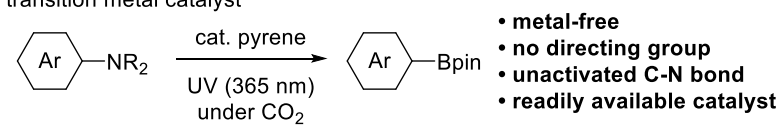

We began our investigation of direct $\mathrm{C}-\mathrm{N}$ bond borylation of $\mathrm{N}, \mathrm{N}$-dimethyl-4-biphenylamine (1a) with 2.0 equiv of $\mathrm{B}_{2}$ pin 2 in the presence of $20 \mathrm{~mol} \%$ pyrene upon photoirradiation at $365 \mathrm{~nm}$ under $\mathrm{N}_{2}$ atmosphere (Table 1). Solvent screening revealed that the desired 4-biphenylboronic acid pinacol ester (2a) was not formed in $\mathrm{CH}_{2} \mathrm{Cl}_{2}, \mathrm{DMF}$, or $\mathrm{MeCN}$ (entries 1-3), but was formed in benzene and ethers (entries 4-7). The best result was obtained with cyclopentyl methyl ether (CPME), and the corresponding product 2 a was obtained in $67 \%$ yield (entry 7 ). ${ }^{28}$ Surprisingly, $\mathrm{CO}_{2}$ atmosphere significantly improved the yield of $\mathbf{2 a}$ to $82 \%$ (entry 8). Moreover, under $\mathrm{CO}_{2}$ atmosphere in a balloon, the yield of 2a reached 93\% (entry 9). Product 2 a was isolated in $80 \%$ yield in a slightly larger scale reaction (entry 10 ). The reaction conducted without the use of pyrene afforded $2 \mathbf{a}$ in low yields both in the presence or absence of $\mathrm{CO}_{2}$ (entries 11 and 12).

Next, we investigated the substrate scope of the aromatic amines using optimal reaction conditions (Scheme 2). To simplify product purification, the resulting aryl boronic acid pinacol esters were transformed to aryl trifluoroborates by treating the reaction mixtures with $\mathrm{KHF}_{2} . \mathrm{N}, \mathrm{N}$-Dimethyl-4biphenylamines with an electron-donating substituent at the para-position of the aniline ring were borylated to afford the corresponding 4-biphenyltrifluoroborate $\mathbf{3 b} \mathbf{b} \mathbf{3 d}$ in good to excellent yields. The cyclopropyl groups of $\mathbf{3 d}$ were tolerated under the reaction conditions. Halogen atoms ( $\mathrm{F}$ and $\mathrm{Cl}$ ) were also left intact, and the corresponding halo-4biphenyltrifluoroborates $\mathbf{3 e}$ and $\mathbf{3 f}$ were produced in high yields. Furthermore, the alkynyl group was tolerated without dimerization or polymerization, and product $\mathbf{3 g}$ was obtained in $72 \%$ yield. The trimethylsilyl group was
Table 1. Optimization of Reaction Conditions

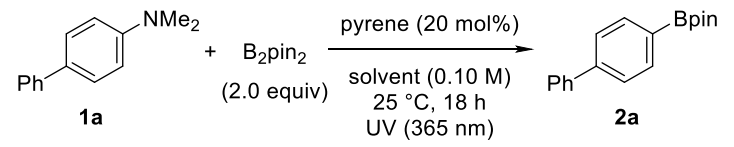

\begin{tabular}{|l|l|l|l|l|}
\hline $\begin{array}{l}\text { en- } \\
\text { trya }\end{array}$ & solvent & atmosphere & conv (\%) $)^{b, c}$ & yield (\%) \\
\hline 1 & $\mathrm{CH}_{2} \mathrm{Cl}_{2}$ & $\mathrm{~N}_{2}$ (close) & 37 & 0 \\
\hline 2 & $\mathrm{DMF}$ & $\mathrm{N}_{2}$ (close) & 49 & 0 \\
\hline 3 & $\mathrm{MeCN}$ & $\mathrm{N}_{2}$ (close) & 68 & 0 \\
\hline 4 & $\mathrm{C}_{6} \mathrm{H}_{6}$ & $\mathrm{~N}_{2}$ (close) & 59 & 50 \\
\hline 5 & $\mathrm{THF}$ & $\mathrm{N}_{2}$ (close) & 80 & 58 \\
\hline 6 & $\begin{array}{l}1,4-\mathrm{di}- \\
\text { oxane }\end{array}$ & $\mathrm{N}_{2}$ (close) & 71 & 66 \\
\hline 7 & $\mathrm{CPME}$ & $\mathrm{N}_{2}$ (close) & 85 & 67 \\
\hline 8 & $\mathrm{CPME}$ & $\mathrm{CO}_{2}$ (close) & 90 & 82 \\
\hline 9 & $\mathrm{CPME}$ & $\mathrm{CO}_{2}$ (balloon) & 97 & 93 \\
\hline $10^{d}$ & $\mathrm{CPME}$ & $\mathrm{CO}_{2}$ (balloon) & 90 & $88(80)^{e}$ \\
\hline $11^{f}$ & $\mathrm{CPME}$ & $\mathrm{N}_{2}$ (close) & 13 & 13 \\
\hline $12^{f}$ & $\mathrm{CPME}$ & $\mathrm{CO}_{2}$ (close) & 15 & 15 \\
\hline
\end{tabular}

a1a $(0.0600 \mathrm{mmol}), \mathrm{B}_{2}$ pin $2(0.120 \mathrm{mmol})$, pyrene $(0.0120$ $\mathrm{mmol})$, CPME $(0.60 \mathrm{~mL}) .{ }^{b}$ Conversion of 1a. ${ }^{c}$ Determined by GC. $d \mathbf{1 a}(0.15 \mathrm{mmol}), \mathrm{B}_{2} \mathrm{pin}_{2}(0.300 \mathrm{mmol})$, pyrene $(0.0300 \mathrm{mmol})$, CPME $(1.5 \mathrm{~mL}) .{ }^{e}$ Isolated yield is described in parentheses. $f$ Without pyrene. THF, tetrahydrofuran; DMF, dimethylformamide; CPME, cyclopentyl methyl ether.

also tolerated under the reaction conditions, giving the corresponding product $\mathbf{2 h}$ in $53 \%$ yield. 4-Mesityl- $N, N$-dimethylaniline with fully twisted aromatic rings or $N, N$-dimethyl-2-amine fluorene-fused aromatic rings were smoothly converted to their corresponding 4-biphenyltrifluoroborate $\mathbf{3 i}$ and fluorenyl trifluoroborate $\mathbf{3 j}$ in $78 \%$ yield and $81 \%$ yield, respectively. The methoxycarbonyl group, which is an electron-withdrawing group, was applied, and the corresponding product $\mathbf{3 k}$ was obtained in $70 \%$ yield. In the case of 2,4-bis(dimethylamino)biphenyl, the $\mathrm{C}-\mathrm{N}$ bond at the 4-position was selectively borylated, giving the corresponding aryl trifluoroborate $\mathbf{3 l}$ in $57 \%$ yield. The borylation of $N, N$-dimethylaniline with a phenyl group at the metaposition also gave product $\mathbf{3 m}$ in $52 \%$ yield, but the borylation of $\mathrm{N}, \mathrm{N}$-dimethyl-2-biphenylamines bearing a substituent at the ortho-position of the aniline ring failed, probably because of steric repulsion (Figure S3). The reaction system was not limited to the biphenyl derivatives, and the deaminative borylation of $\mathrm{N}, \mathrm{N}$-dimethyl-4-(thiophen-2-yl)aniline afforded the borylated product $3 \mathbf{n}$ in $54 \%$ yield. In addition, $N, N$-dimethyl-cyclohexenylaniline and $N, N$-dimethyl-naphthylamines were successfully transformed into the corresponding aryl trifluoroborate 3o-3q. 
Scheme 2. Direct Borylation of Aromatic Amines by Cleavage of Unactivated C-N Bond

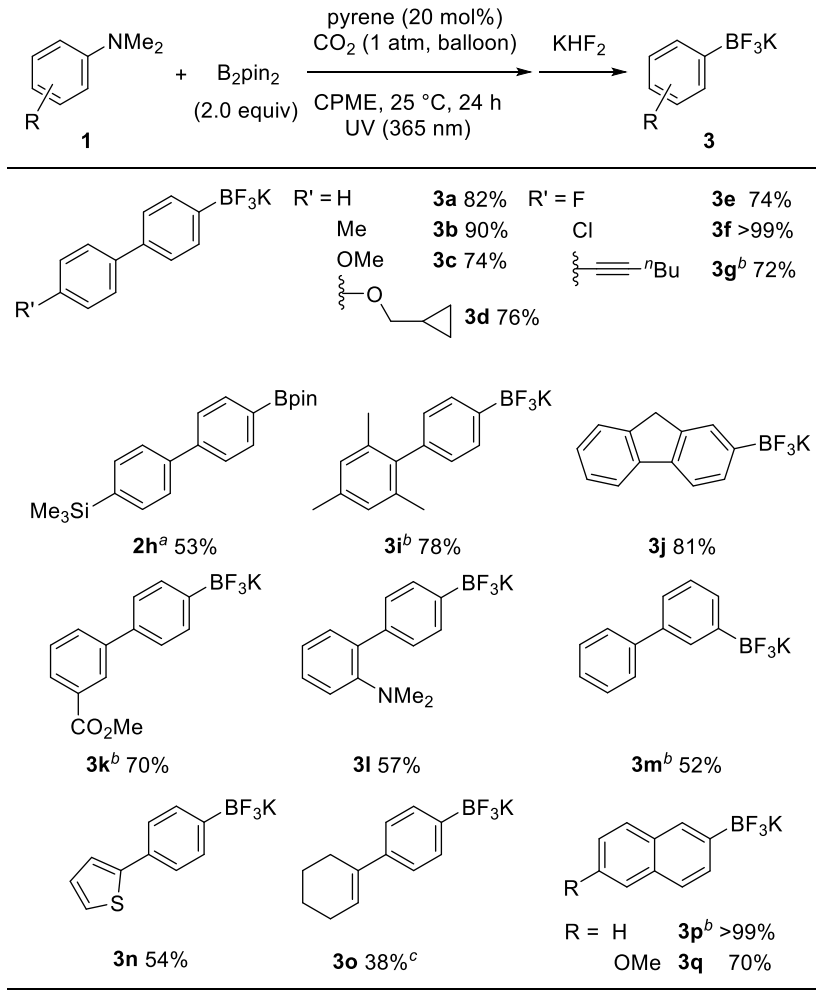

a Isolated yield as pinacol ester without the treatment with $\mathrm{KHF}_{2}$ is described in parentheses. ${ }^{b} \mathrm{~B}_{2}$ pin 2 (3.0 equiv), pyrene (30 mol\%), 36 h. ${ }^{c} 12 \mathrm{~h}$.

Next, the borylation of biphenylamines bearing different substituents on the nitrogen atom was investigated (Scheme 3). The reaction system was applied to cyclic amines with pyrrolidyl or piperidyl groups, 1r and 1s, to afford trifluoroborate $3 a$ in $46 \%$ and $75 \%$ yields, respectively. Surprisingly, the borylation of $N$-methylamine 1 t proceeded to give $\mathbf{3 a}$ in $77 \%$ yield. ${ }^{30,31}$

\section{Scheme 3. Direct Borylation of Aromatic Amines with Other Amino Groups}
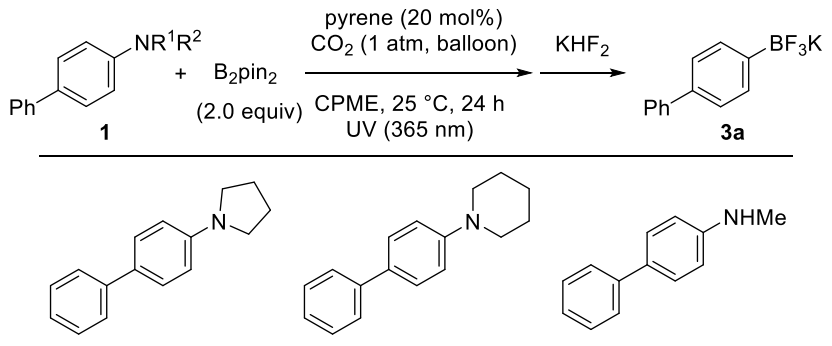

1r $46 \%$

1s $75 \%^{a}$

1t $77 \%$ that the $\mathrm{C}-\mathrm{N}$ bond cleavage in anilines did not proceed via a radical intermediate. ${ }^{32,33}$ To investigate our hypothesis, borylation was conducted in the presence of 1,1-diphenylethylene or 2,2,6,6-tetramethylpiperidine 1-oxyl (TEMPO) as a radical trapping reagent (Scheme 4). Therefore, the borylation of 1a proceeded smoothly and product 2a was obtained in $81 \%$ and $62 \%$ yields, respectively. Furthermore, radical addition products derived from arylamine 1a were not observed by GC-MS (Scheme 4). ${ }^{34}$ In the reaction with 10 equiv of $\mathrm{MeOH}, 2$ a was obtained in $46 \%$ yield, but Ar-OMe was not detected at all (Table S7, entry 5). The results indicated that the borylation did not involve generation of $\alpha$-amino methyl radicals, aryl radicals, or aryl cations, which suggested that both the $\mathrm{C}-\mathrm{N}$ bond cleavage and the $\mathrm{C}-\mathrm{B}$ bond formation presumably proceeded via a concerted mechanism.

Scheme 4. Borylation Reactions in the Presence of Radical Trap Reagent

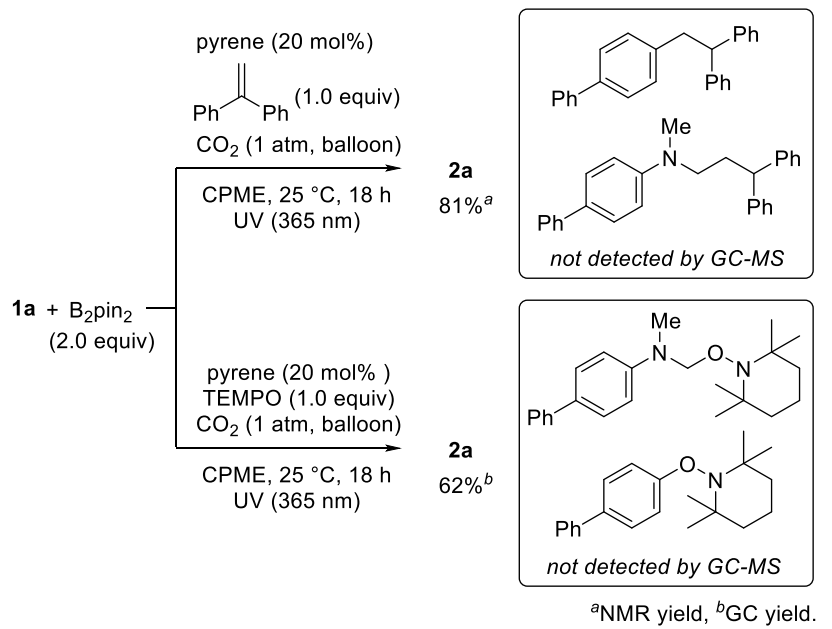

Next, the rate orders of each component (1a, $\mathrm{B}_{2} \mathrm{pin}_{2}$, and pyrene) were determined by kinetic analyses (Figures S68).$^{35}$ The reaction rates were found to be first-order in both 1a and $\mathrm{B}_{2} \mathrm{pin}_{2}$ (Figures S6 and S7). Interestingly, a negative correlation between pyrene concentration and initial reaction rate was observed (Figure S8). This is probably due to pyrene excimer formation at high concentrations of pyrene, which suppresses electron transfer via the pyrene monomer. ${ }^{36}$

To gain insight into the reaction mechanism, we conducted an NMR experiment on the borylation of 1a, $\mathrm{B}_{2}$ pin $_{2}$ (1.0 equiv), and pyrene (20 mol\%) in THF- $d_{8}$ under $\mathrm{CO}_{2}$ atmosphere (Scheme 5). As a result, two new signals at 20.3 ppm and $22.9 \mathrm{ppm}$ were observed in the ${ }^{11} \mathrm{~B}$ NMR spectrum, which were assigned to $\mathbf{2 a}$ and $N, N$-dimethylaminopinacolborane (pinB-NMe $)_{2},{ }^{37}$ respectively (Scheme 5). The results proved that borylation proceeded and pinB-NMe $\mathrm{N}_{2}$ was generated as a byproduct.

We next initiated mechanistic studies to elucidate the reaction mechanism of direct $\mathrm{C}-\mathrm{N}$ bond borylation. The tolerance of cyclopropyl group of $\mathbf{3 d}$ under the reaction conditions, as shown in Scheme 2, can be attributed to the fact 
Scheme 5. Observation of pinB-NMe2 Species by ${ }^{11} \mathrm{~B}$ NMR Experiment

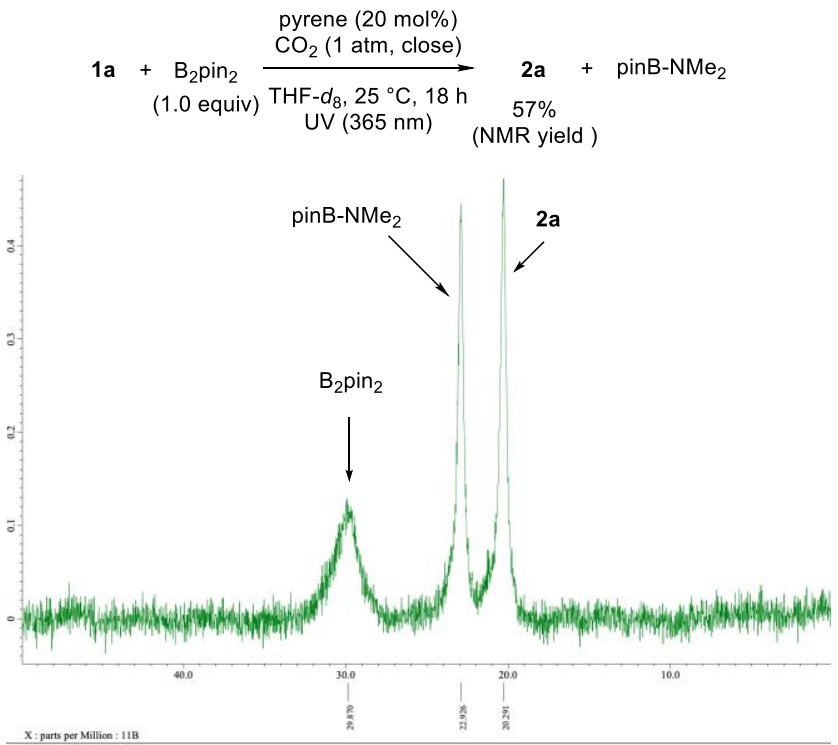

Control experiments were conducted (Scheme 6) to investigate whether the gradual generation of pinB- $\mathrm{NMe}_{2}$ inhibited the borylation of 1a. Indeed, the addition of 1.0 equiv of pinB- $\mathrm{NMe}_{2}$ into the reaction system under $\mathrm{N}_{2}$ atmosphere decreased the yield of $2 \mathrm{a}$ from $67 \%$ (Table 1, entry 1) to $35 \%$. This result indicated that pinB- $\mathrm{NMe}_{2}$ acts as a reaction inhibitor. Next, we investigated the effect of $\mathrm{CO}_{2}$ on the reaction; we assumed that $\mathrm{CO}_{2}$ weakened the inhibitory effect of pinB-NMe $\mathrm{N}_{2}$. Based on the above assumption, borylation with 1.0 equiv of pinB-NMe2 under $\mathrm{CO}_{2}$ atmosphere was conducted, resulting in a higher yield than that under $\mathrm{N}_{2}$ atmosphere (Scheme 6). To investigate its effect, the borylations under $\mathrm{N}_{2}$ and $\mathrm{CO}_{2}$ atmospheres were monitored (Figure S11). Initial reaction rates show a similar tendency, whereas at a later stage, the reaction under $\mathrm{N}_{2}$ atmosphere was slower than that under $\mathrm{CO}_{2}$ atmosphere. This result suggested that $\mathrm{CO}_{2}$ reduced the inhibition by pinB$\mathrm{NMe}_{2}{ }^{38}$ rather than activated aromatic amines, which agrees with the result that the pyrene-free reactions with or without $\mathrm{CO}_{2}$ afforded similar product yields (Table 1, entries 11 and 12).

Scheme 6. Examinations of Inhibition Effect by pinB$\mathrm{NMe}_{2}$

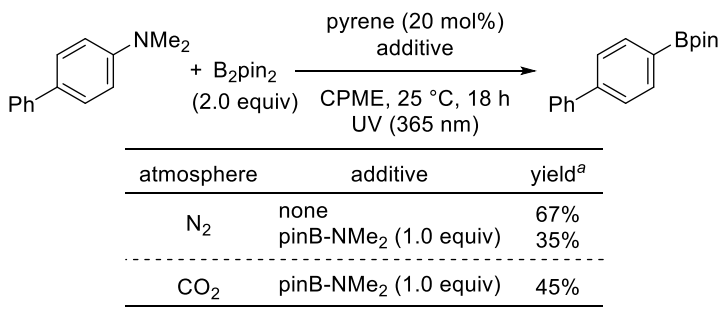

${ }^{a}$ Determined by GC.

The proposed mechanism for C-N borylation, derived from the above mechanistic studies, is depicted in Scheme 7: (1) photoinduced single electron transfer from the excited pyrene occurs upon UV light photoexcitation to form a complex of the arylamino radical cation with $\mathrm{B}_{2} \mathrm{pin}_{2}$; (2) C-N bond cleavage C-B bond formation of the resulting radical cation species occur by a concerted pathway; the aminoboryl radical cation generates as a byproduct; and (3) electron transfer from the pyrene radical anion to the aminoboryl radical cation resulting in pyrene and aminoborane, which might interact with $\mathrm{CO}_{2}$.

\section{Scheme 7. Proposed Reaction Mechanism}

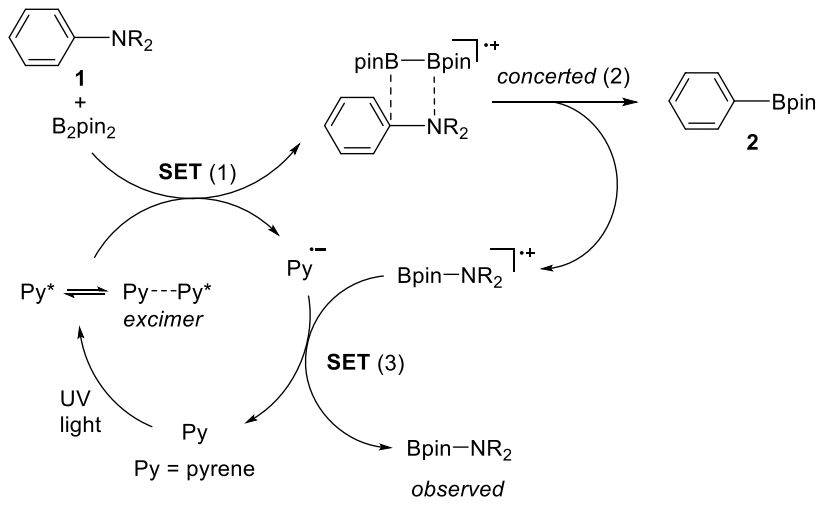

In summary, to the best of our knowledge, this is the first study to develop a photoinduced metal-free direct borylation of aromatic amines via unactivated $\mathrm{C}-\mathrm{N}$ bonds cleavage. We found that the yields of the borylated products improved dramatically under $\mathrm{CO}_{2}$ atmosphere. The reaction system was applied to various aromatic amines, efficiently producing the corresponding borylated products. In the mechanistic study, the demonstration of radical trap experiments suggested that both the $\mathrm{C}-\mathrm{N}$ bond cleavage and the $\mathrm{C}-\mathrm{B}$ bond formation proceeded via a concerted mechanism. Moreover, the results of the ${ }^{11} \mathrm{~B}$ NMR experiment and the reactions in the presence of aminoborane, which was a byproduct, indicated that aminoborane had an inhibitory effect, which was reduced by $\mathrm{CO}_{2}$. The elucidation of a more detailed reaction mechanism is underway in our group. We believe that this study will shed light on a new strategy for transformation via of unactivated $\mathrm{C}-\mathrm{N}$ bonds cleavage.

\section{ASSOCIATED CONTENT}

Supporting Information.

The Supporting Information is available free of charge via the Internet at http://pubs.acs.org.

\section{AUTHOR INFORMATION}

\section{Corresponding Authors}

Kohei Sekine - Institute for Materials Chemistry and Engineering, Kyushu University, 6-1 Kasugakoen, Kasuga-shi, Fukuoka 816-8580, Japan, and Interdisciplinary Graduate School of Engineering Sciences, Kyushu University, 6-1 Kasugakoen, Kasuga-shi, Fukuoka 816-8580, Japan; orcid.org/0000-0001-7588-3176; Email: sekine@cm.kyushu-u.ac.jp

Yoichiro Kuninobu - Institute for Materials Chemistry and Engineering, Kyushu University, 6-1 Kasugakoen, Kasuga-shi, Fukuoka 816-8580, Japan, and Interdisciplinary Graduate School of Engineering Sciences, Kyushu University, 6-1 Kasugakoen, 
Kasuga-shi, Fukuoka 816-8580, Japan; orcid.org/0000-00028679-9487; E-mail: kuninobu@cm.kyushu-u.ac.jp

\section{Author}

Akira Shiozuka - Interdisciplinary Graduate School of Engineering Sciences, Kyushu University, 6-1 Kasugakoen, Kasuga-shi, Fukuoka 816-8580, Japan

\section{Notes}

The authors declare no competing financial interest.

\section{ACKNOWLEDGMENT}

This work was supported in part by JSPS KAKENHI Grant Numbers JP 17H03016, 18H04656, and 20H04824, Takeda Science Foundation, The Mitsubishi Foundation, and Yamada Science Foundation. This work was the result of using research equipments shared in MEXT Project for promoting public utilization of advanced research infrastructure (Program for supporting introduction of the new sharing system) Grant Number JPMXS0422300120.

\section{REFERENCES}

(1) Luo, Y.-R. Handbook of Bond Dissociation Energies in Organic Compounds. CRC Press, 2002.

(2) Wang, Z.-X.; Yang, B. Chemical transformations of quaternary ammonium salts via C-N bond cleavage. Org. Biomol. Chem. 2020 18, 1057-1072.

(3) Zhang, H.; Hagihara, S.; Itami, K. Making Dimethylamino a Transformable Directing Group by Nickel-Catalyzed C-N Borylation. Chem. Eur. J. 2015, 21, 16796-16800.

(4) Mo, F.; Jiang, Y.; Qiu, D.; Zhang, Y.; Wang, J. Direct Conversion of Arylamines to Pinacol Boronates: A Metal-Free Borylation Process. Angew. Chem., Int. Ed. 2010, 49, 1846-1849.

(5) Qiu, D.; Jin, L.; Zheng, Z.; Meng, H.; Mo, F.; Wang, X.; Zhang, Y.; Wang, J. Synthesis of Pinacol Arylboronates from Aromatic Amines: A Metal-Free Transformation. J. Org. Chem. 2013, 78, 1923-1933.

(6) Yang Z.-K.; Xu, N.-X.; Wang, C.; Uchiyama, M. Photoinduced $\mathrm{C}\left(\mathrm{sp}^{3}\right)-\mathrm{N}$ Bond Cleavage Leading to the Stereoselective Syntheses of Alkenes. Chem. Eur. J. 2019, 25, 5433-5439.

(7) Sandfort, F.; Strieth-Kalthoff, F.; Klauck, F. J. R.; James, M. J.; Glorius, F. Deaminative Borylation of Aliphatic Amines Enabled by Visible Light Excitation of an Electron Donor-Acceptor Complex. Chem. Eur. J. 2018, 24, 17210-17214.

(8) Ma, Y.; Pang, Y.; Chabbra, S.; Reijerse, E. J.; Schnegg, A.; Niski, J.; Leutzsch, M.; Cornella, J. Radical C-N Borylation of Aromatic Amines Enabled by a Pyrylium Reagent. Chem. Eur. J. 2020, 26, 3738-3743.

(9) Wu, J.; He, L.; Noble, A.; Aggarwal, V. K. Photoinduced Deaminative Borylation of Alkylamines. J. Am. Chem. Soc. 2018, 140, 10700-10704.

(10) Tobisu, M.; Nakamura, K.; Chatani, N. Nickel-Catalyzed Reductive and Borylative Cleavage of Aromatic Carbon-Nitrogen Bonds in N-Aryl Amides and Carbamates. J. Am. Chem. Soc. 2014, 136, 5587-5590.

(11) Zhang, Z.-B.; Xia, J. B. NHC-Nickel Catalyzed C-N Bond Cleavage of Mono-protected Anilines for C-C Cross-Coupling. Org. Lett. 2020, 22, 9609-9613.

(12) Zhang, Z. B.; Ji, C. L.; Yang, C.; Chen, J.; Hong, X.; Xia, J. B. NickelCatalyzed Kumada Coupling of Boc-Activated Aromatic Amines via Nondirected Selective Aryl C-N Bond Cleavage. Org. Lett. 2019, 21, 1226-1231.

(13) Ueno, S.; Chatani, N.; Kakiuchi, F. Ruthenium-Catalyzed Carbon-Carbon Bond Formation via the Cleavage of an Unreactive Aryl Carbon-Nitrogen Bond in Aniline Derivatives with Organoboronates. J. Am. Chem. Soc. 2007, 129, 6098-6099.

(14) Koreeda, T.; Kochi, T.; Kakiuchi, F. Cleavage of C-N Bonds in Aniline Derivatives on a Ruthenium Center and Its Relevance to
Catalytic C-C Bond Formation. J. Am. Chem. Soc. 2009, 131, 71387239.

(15) Koreeda, T.; Kochi, T.; Kakiuchi, F. Ruthenium-catalyzed reductive deamination and tandem alkylation of aniline derivatives. J. Organomet. Chem. 2013, 741-742, 148-152.

(16) Tang, J.; Fan, F.; Cong, X.; Zhao, L.; Luo, M.; Zeng, X. Reductive Cross-Coupling between Unactivated $\mathrm{C}(\operatorname{aryl})-\mathrm{N}$ and $\mathrm{C}(\operatorname{aryl})-\mathrm{O}$ Bonds by Chromium Catalysis Using a Bipyridyl Ligand. J. Am. Chem. Soc. 2020, 142, 12834-12840.

(17) Cong, X.; Fan, F.; Ma, P.; Luo, M.; Chen, H.; Zeng, X. Low-Valent, High-Spin Chromium-Catalyzed Cleavage of Aromatic Carbon-Nitrogen Bonds at Room Temperature: A Combined Experimental and Theoretical Study. J. Am. Chem. Soc. 2017, 139, 15182-15190. (18) Xu, J.-X.; Zhao, F.; Yuan, Y.; Wu, X.-F. Ruthenium-Catalyzed Carbonylative Coupling of Anilines with Organoboranes by the Cleavage of Neutral Aryl C-N Bond. Org. Lett. 2020, 22, 2756-2760.

(19) Xu, J.-X.; Zhao, F.; Franke, R.; Wu, X.-F. Ruthenium-catalyzed Suzuki coupling of anilines with alkenyl borates via selective aryl C-N bond cleavage. Catal. Commun. 2020, 140, 106009.

(20) Cao, Z.-C.; Li, X.-L.; Luo, Q.-Y.; Fang, H.; Shi, Z.-J. Direct Borylation of Tertiary Anilines via $\mathrm{C}-\mathrm{N}$ Bond Activation. Org. Lett. 2018, 20, 1995-1998.

(21) Cao, Z.-C.; Xie, S.-J.; Fang, H.; Shi, Z.-J. Ni-Catalyzed Cross-Coupling of Dimethyl Aryl Amines with Arylboronic Esters under Reductive Conditions, J. Am. Chem. Soc. 2018, 140, 13575-13579.

(22) Wang, Q.; Su, Y.; Li, L.; Huang, H. Transition-metal catalysed C$\mathrm{N}$ bond activation. Chem. Soc. Rev. 2016, 45, 1257-1272.

(23) Ouyang, K.; Hao, W.; Zhang, W.-X.; Xi, Z. Transition-Metal-Catalyzed Cleavage of C-N Single Bonds. Chem. Rev. 2015, 115, 12045-12090.

(24) Friese, F. W.; Studer, A. New avenues for C-B bond formation via radical intermediates. Chem. Sci. 2019, 10, 8503-8518.

(25) Wang, M.; Shi, Z. Methodologies and Strategies for Selective Borylation of C-Het and C-C Bonds. Chem. Rev. 2020, 120, 73487398.

(26) Miyaura, N.; Suzuki, A. Palladium-Catalyzed Cross-Coupling Reactions of Organoboron Compounds. Chem. Rev. 1995, 95, $2457-$ 2483.

(27) Ghosh, I.; Shaikh, R. S.; König, B. Sensitization-Initiated Electron Transfer for Photoredox Catalysis. Angew. Chem., Int. Ed. 2017, $56,8544-8549$.

(28) Lu, J.; Khetrapal, N. S.; Johnson, J. A.; Zeng, X. C.; Zhang, J. " $\pi-$ Hole- $\pi$ " Interaction Promoted Photocatalytic Hydrodefluorination via Inner-Sphere Electron Transfer. J. Am. Chem. Soc. 2016, 138, 15805-15808.

(29) For the screening of other photosensitizers, such as benzophenone and 9,10-diphenylanthracene, see Table S4 in the Supporting Information.

(30) It is significant that the borylation of 4-aminobiphenyl also proceeded to give the borylated product 3a whereas the yield was low $(16 \%$ GC yield).

(31) The borylation of $N$-biphenylpyrrole used in the previous literature reported by Shi group ${ }^{20}$ was failed. The reaction conditions were not suitable to substrates bearing steric congested amino groups and moderate-to-strong electron-withdrawing substituents. See Figure S3.

(32) The reaction of $N, N$-dimethylaniline excited by a photosensitizer generally includes the formation of $\alpha$-amino radical intermediate. See: Yang, S.; Li, P.; Wang, Z.; Wang, L. Photoinduced Oxidative Formylation of $N, N$-Dimethylanilines with Molecular Oxygen without External Photocatalyst. Org. Lett. 2017, 19, 3386-3389.

(33) Aramaki, Y.; Imaizumi, N.; Hotta, M.; Kumagai, J.; Ooi, T. Exploiting single-electron transfer in Lewis pairs for catalytic bondforming reactions. Chem. Sci. 2020, 11, 4305-4311.

(34) The borylated compound of 1,1-diphenylethylene was obtained in $65 \%$ yield. See the Supporting Information for the detail (Scheme S1)

(35) See the Supporting Information for exact details of reaction conditions. 
(36) The Stern-Volmer experiments suggested that a quench of excited pyrene monomer $(\lambda=394 \mathrm{~nm})$ is more favorable than that of pyrene excimer $(\lambda=480 \mathrm{~nm})$. See Figures S9 and S10.

(37) Solé, C.; Fernundez, E. Alkoxide Activation of Aminoboranes towards Selective Amination. Angew. Chem., Int. Ed. 2013, 52, 11351-11355.
(38) We assumed that an adduct between $\mathrm{CO}_{2}$ and pinB- $\mathrm{NMe}_{2}$ formed, or pinB- $\mathrm{NMe}_{2}$ was transformed by $\mathrm{CO}_{2}$. However, it is not clear so far. For the transformation of aminoboranes with $\mathrm{CO}_{2}$, see: Cragg, R. H.; Lappert, M. F.; Niith, H.; Schweizer, P.; Tilley, B. P. Die Aminoborierung von Kohlendioxid und Kohlenstoffdisulfid. Chem. Ber. 1967, 100, 2377-2382. 


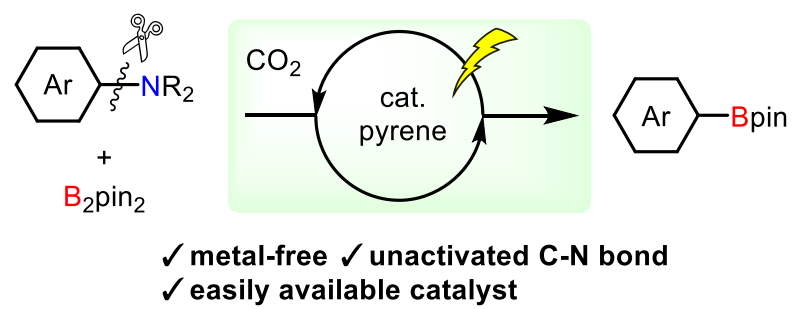

